\title{
The 4-H of Biomarkers in Arthritis: A Lot of Help, Occasional Harm, Some Hype, Increasing Hope
}

\author{
Gilles Boire and Hugues Allard-Chamard
}

\begin{abstract}
Gilles Boire): It was both a pleasure and an honor to present the 2019 Dunlop-Dottridge Lecture. My co-author and I will now discuss benefits and pitfalls of biomarkers developed through emerging techniques, evaluated through the experiential perspective of a seasoned clinician, as they apply to the quest for biomarker identification in rheumatic diseases. (J Rheumatol 2019;46:758-63; doi:10.3899/jrheum.190375)
\end{abstract}

\author{
Key Indexing Terms: \\ BIOLOGICAL MARKERS \\ ENVIRONMENT
}

\section{GENETIC PREDISPOSITION TO DISEASE AUTOIMMUNE DISEASES OUTCOMES}

Thousands of papers on biomarkers in rheumatology are published each year, and the numbers are increasing steadily. After decades of relatively simple biomarkers such as autoantibodies and single proteins, we are on the verge of a revolution that is already transforming other areas of medicine: the availability of next-generation biomarkers resulting from the combination of high throughput techniques allowing the collection of thousands to millions of variables at the same time, and their simultaneous analysis by computational tools, yielding various scores, signatures, or subsets to be used for pathogenic studies, biologically based diagnosis, prognosis, and selection of optimal drug regimens. While heralding a new era in rheumatology, these complex biomarkers raise challenges and risks that need to be carefully addressed before their implementation. We will first concentrate on the uses and misuses of current biomarkers to illustrate how the next generation of composite biomarkers will positively and unfortunately, potentially negatively alter our approach to disease pathogenesis, diagnosis, and treatments.

\section{The ABC of Biomarkers}

Biomarkers are objectively measured characteristics that evaluate physiological or pathogenic processes, as well as potential indicators of therapeutic responses ${ }^{1}$. Variables

From the Department of Medicine, Faculty of Medicine and Health Sciences, Université de Sherbrooke; Department of Medicine, Centre intégré universitaire de Santé et de Services Sociaux de l'Estrie - Centre Hospitalier Universitaire de Sherbrooke (CIUSSS de l'Estrie-CHUS), Sherbrooke, Quebec, Canada.

H. Allard-Chamard, MD, PhD, Department of Medicine, Faculty of Medicine and Health Sciences, Université de Sherbrooke, and Department of Medicine, CIUSSS de l'Estrie-CHUS, currently Research Fellow in Medicine, Ragon Institute of Massachusetts General Hospital, Massachusetts Institute of Technology, and Department of Medicine, Harvard University; G. Boire, MD, MSc, Department of Medicine, Faculty of Medicine and Health Sciences, Université de Sherbrooke, and Department of Medicine, CIUSSS de l'Estrie-CHUS.

Address correspondence to Dr. G. Boire, 3001 12th Ave. North, Division of Rheumatology, Room 3853, Sherbrooke, Quebec J1H 5N4, Canada. E-mail: gilles.boire@usherbrooke.ca indicative of a patient's feelings, well-being, or functions, are not considered biomarkers. A biomarker may consist of a single variable or be composite when exploiting input from multiple variables ${ }^{2}$. In rheumatology, biomarkers contribute to various clinical objectives (Table 1$)^{3}$.

Biomarkers may give mechanistic, clinical, or therapeutic information ${ }^{4}$. They come under various forms, such as proteins (frequently antibodies), genetic and epigenetic traits, imaging results, histological findings, cellular responses, gene expression, or microbiome characterization ${ }^{3}$. Their sources are diverse, ranging from fluids (blood, serum, plasma, urine, saliva, synovial fluid), isolated blood cells, skin, gums, membranes and organs, feces, imaging, and even digital (such as those obtained from a smart watch) ${ }^{3}$.

The ideal biomarker is safe and easy to measure, sensitive and specific, reproducible, consistent across gender and race, actionable such that it can inform clinical management, and cost-efficient ${ }^{3,4}$. Many biomarkers are co-correlated and thus their concomitant usage does not add much to the information generated by a single one [e.g., erythrocyte sedimentation rate and $\mathrm{C}$-reactive protein (CRP) to evaluate $\mathrm{RA}$ disease activity].

\section{Current Uses (HELP) and Misuses (HARM) of Biomarkers}

Current biomarkers are best used to support clinical decisions, especially to support or invalidate hypotheses resulting from careful collection of signs and symptoms. Antibodies are often considered the rheumatologists' area of expertise. It is thus appropriate to use them to illustrate the good and the bad sides of current biomarkers.

- HELP

For example, a 72-year-old woman is referred for presumed polymyalgia rheumatica. She presents with a few weeks of shoulder and hip pain and limitation, the CRP is increased, but you note swelling and tenderness in some small joints of both hands and in wrists. You think of rheumatoid arthritis (RA). If anti-CCP [anticyclic citrullinated peptide] and

Personal non-commercial use only. The Journal of Rheumatology Copyright @ 2019 . All rights reserved. 
Table 1. Current uses of biomarkers in rheumatology.

\begin{tabular}{|c|c|}
\hline Purpose & Examples of Biomarkers \\
\hline Susceptibility/risk factor assessment & HLA-B27, smoking, obesity \\
\hline Diagnostics & $\begin{array}{l}\text { Periarticular erosions in at least } 3 \text { joints in a patient with } \\
\text { arthritis to diagnose RA; presence of intracellular sodium } \\
\text { urate crystals in polymorphonuclear cells during } \\
\text { synovioanalysis to diagnose gout }\end{array}$ \\
\hline Monitoring & $\begin{array}{l}\text { CRP during polymyalgia rheumatica treatment; swollen joint } \\
\text { counts in RA patients }\end{array}$ \\
\hline Prognostics (relates to natural history of the disease) & $\begin{array}{l}\text { High-titer anti-CCP antibodies; persistent depressive } \\
\text { symptoms in RA patients }\end{array}$ \\
\hline $\begin{array}{l}\text { Predictive (relates to benefit or harm from a specific } \\
\text { therapy) }\end{array}$ & $\begin{array}{l}\text { TPMT genotype and risk of azathioprine toxicity; } \\
\text { seropositivity in RA patients and degree of response to } \\
\text { rituximab }\end{array}$ \\
\hline Pharmacodynamic/response & Blood levels of anti-TNF drugs; CRP \\
\hline Safety & ALT levels; neutrophil counts \\
\hline Surrogate endpoints & $\begin{array}{l}\text { Bone densitometry changes and prediction of fragility } \\
\text { fracture risk; remission according to SDAI and prevention of } \\
\text { progression of radiographic erosions in RA }\end{array}$ \\
\hline Development of drug target & Cytokine expresion in serum and synovial membrane \\
\hline
\end{tabular}

RA: rheumatoid arthritis; CRP: C-reactive protein; CCP: cyclic citrullinated peptide; TPMT: thiopurine methyltransferase; TNF: tumor necrosis factor; ALT: alanine aminotransferase; SDAI: Simple Disease Activity Index.

rheumatoid factor are both strongly positive, you feel very comfortable with the diagnosis.

Similarly, seeing a 68-year-old man with a few weeks of fatigue and altered general health now presenting with severe pulmonary hemorrhage and rapidly worsening kidney failure, you immediately think of vasculitis. A diagnosis of double-positive Goodpasture syndrome based on the presence of both positive antiglomerular basement membrane and antimyeloperoxidase antibodies will prompt the initiation of both plasmapheresis and immunosuppression in an attempt to save the patient's life.

\section{- HARM}

When biomarkers take precedence over the clinical presentation, problems arise. Every rheumatologist has seen inappropriate referrals for patients with lower back pain and a positive ANA (antinuclear antibody), or with generalized pain typical of fibromyalgia and a positive anti-CCP. For sure, positive biomarkers may represent a preclinical warning for future disease development, but without appropriate clinical signs and symptoms, they mostly generate unnecessary anxiety and potential harm, without benefits to the patient. Conversely, the absence of positive biomarkers may also pose a significant hurdle to establishing a correct diagnosis. For example, despite persistent distal symmetrical synovitis, the diagnosis of RA may be delayed in the absence of RA-associated antibodies together with a normal CRP.

Sometimes biomarkers lead one to consider the wrong diagnosis. For example, in a 52-year-old woman with fever, weight loss, purpura, proteinuria, and progressive renal insufficiency developing over several weeks, negative blood cultures and positive ANCA (antineutrophil cytoplasmic antibodies) and anti-PR3 antibodies suggest ANCA-associ- ated vasculitis (AAV). However, if the patient also has a heart murmur and low complement levels, features not typical of $\mathrm{AAV}$, alternative diagnoses need to be actively looked for. Circulating Bartonella henselae DNA identified by PCR, a diagnosis later confirmed by serology, fully explains the clinical picture; false-positive ANCA and anti-PR3 are indeed frequent in this infection ${ }^{5}$. In this case, immunosuppression instead of antibiotherapy would have been detrimental. It is thus critical to carefully account for all clinical findings when interpreting biomarkers' results 6 .

Remarkably, biomarkers also have frequently ignored inherent limitations. The first and most obvious is that biomarkers do not holistically represent the actual patients. Indeed, when one wants to identify patients with recent-onset inflammatory polyarthritis who will reach remission over the following 5 years, biomarkers are of little use, while elevated scores of depressive symptoms offer some pertinent information ${ }^{7}$.

The value of a biomarker may also change over time. Collectively, we have learned, and teach that seropositive RA is more severe than seronegative. However, now that we treat patients earlier and more intensively aiming to remission, outcomes at 1 year are very similar regardless of seropositivity $^{8}$. Unrelated changes in practice may thus alter the course of disease and make some biomarkers less pertinent; similarly, when a biomarker becomes largely used, it induces changes in diagnosis and treatment that may blunt its original impact on prognosis.

Moreover, some biomarkers may lead to rigid and potentially counterproductive conceptualizations. For example, tentative models of RA pathogenesis through RA-associated antibodies $^{9,10}$ fail to synthesize all available information, such as data in Table $2^{11}$. Baseline variables from these 754

Personal non-commercial use only. The Journal of Rheumatology Copyright (C) 2019. All rights reserved. 
Table 2. Baseline characteristics in 754 consecutive RA patients from the Early Undifferentiated PolyArthritis (EUPA) cohort.

\begin{tabular}{lccc}
\hline Baseline Variables & Anti-CCP2-negative, $\mathrm{n}=462$ & Anti-CCP2-positive, $\mathrm{n}=272$ & $\mathrm{p}$ \\
\hline Age, $\mathrm{yrs} \#$ & $65.0(52.8-74.1)$ & $58.2(46.8-66.5)$ & $<0.0001$ \\
Women* & $280(60.6)$ & $159(58.5)$ & 0.5661 \\
Body mass index, $\mathrm{kg} / \mathrm{m}^{2 \#}$ & $26.6(23.1-30.0)$ & $26.2(23.1-29.5)$ & 0.7168 \\
Active smokers* & $69(15.5)$ & $58(21.8)$ & 0.0350 \\
Nonsmokers (ever)* & $180(40.5)$ & $3.8(2.3-7.0)$ & 0.0220 \\
Duration of symptoms, mos $^{\#}$ & $3.4(1.9-5.6)$ & $137(62.0)$ & 0.0004 \\
Shared epitope (SE) alleles* & $118(33.8)$ & $<.0001$ \\
\hline
\end{tabular}

${ }^{\#}$ Median (25th and 75th percentiles). * Number (\%). SE in local population: 38\%. RA: rheumatoid arthritis; anti-CCP: anticyclic citrullinated peptide antibodies.

consecutive RA patients enrolled into our EUPA (Early Undifferentiated PolyArthritis) cohort are typical of very early $\mathrm{RA}^{8,12}$. Seronegative and seropositive patients had quite similar clinical presentation; their smoking history was alike; one-third of anti-CCP-positive patients did not bear any HLA-DR shared epitope. Further, over the course of the first 5 years of disease, OR for bony erosions was only 1.5 in seropositives versus seronegatives, and the use of biologic DMARD (disease-modifying antirheumatic disease) was numerically higher, but not significantly (not shown). Clearly, antibodies alone do not define nonoverlapping subsets of RA patients according to their clinical presentation, pathogenic process, environmental exposures, or outcomes.

\section{Next-generation Computational Biomarkers}

We are entering an era of computational biomarkers originating from data generated by ever more efficient molecular technologies allowing multiplexing and generation of minimally biased biomarkers. Low-cost DNA sequencing allows for full genome sequencing of an individual ${ }^{13}$, as well as the determination of species of bacteria in a stool specimen, without the need for culture ${ }^{14}$. Gene expression can be determined in hundreds of individual cells, showing surprising diversity within a single population of cells ${ }^{15}$. Proteomic techniques can detect simultaneously hundreds of proteins in a single sample ${ }^{16}$. Epigenetics is revealing the crucial role of histone modifications, noncoding RNA, and DNA methylation in cell fate and functions ${ }^{17}$. Making sense of such a large volume of data requires advanced statistical methods and techniques to explore and learn the unknown internal structure of big data. These techniques encompass machine learning, neural networks, and clustering techniques without a priori assumptions such as Distributed Stochastic Neighbour Embedding, Uniform Manifold Approximation and Projection, and Principal Component Analysis (PCA). While signatures generated using these methods appear simple, clinicians are not equipped to apprehend how complex these computer-generated biomarkers are and what they mean. The ultimate objective of these composite biomarkers is "systems medicine," integrating biochemical, physiological, and environmental interactions ${ }^{18}$. Yet questions remain: will patients benefit from this techno- logy-driven explosion of biomarkers and do we prepare adequately for them?

\section{Next-generation Biomarkers: HOPE and HYPE}

- Response to treatment

Today, predicting response to MTX (methotrexate) remains impossible. Clinical covariates such as age, smoking status, antibodies, joint counts, and patient evaluations only explained $63 \%$ of the area under the receiver operating characteristics (ROC) curve (AUC) to predict MTX nonresponse $^{19}$. However, gene expression profiles from whole blood RNA showed an overrepresentation of type I interferon pathway genes in nonresponders. This signature explained $78 \%$ of the AUC, a significant improvement over clinical assessment $^{19}$. Recently, combining clinical and genomic variables (specifically, genotypes of adenosine triphosphatebinding cassette transporter implicated in active MTX efflux from cells) yielded a model with an AUC of $80 \%{ }^{20}$, illustrating how various sources of biomarkers can synergize productively.

- Disease pathogenesis (HOPE)

There is also hope that having more in-depth biological information will give clues about pathogenesis, subsequently allowing for a more uniform classification of patients and informing treatment options.

We propose using juvenile idiopathic arthritis (JIA) as an example. Current International League of Associations for Rheumatology (ILAR) classification of JIA into 7 subtypes is based on the number of joints affected over the first 6 months, combined with biomarkers and extraarticular manifestations ever present in the patient or in first-degree relatives $^{21}$. Patients from the same subtype vary in clinical presentations, response to treatments, and outcomes (e.g., remission). Our Canadian pediatric colleagues reported their preliminary observations using 2 cohorts, ReACCh Out (Research in Arthritis in Canadian Children Emphasizing Outcomes) and BBOP (Biologically-Based Outcome Predictors in JIA) ${ }^{22}$. Using PCA statistical methods to analyze serum cytokine and chemokine expression combined with clinical and biologic variables, they reported 5 patient clusters more homogeneous than ILAR subtypes, both in clinical presentation and outcomes at 6 months.

Personal non-commercial use only. The Journal of Rheumatology Copyright (C) 2019. All rights reserved. 
Similar findings were reported for pediatric SLE (systemic lupus erythematosus) using gene signatures from whole blood RNA-Sequencing ${ }^{23}$. Seven SLE subgroups with specific combinations of 5 immune signatures were found.

Biologically based disease delineation is still in its infancy. Limitations of the current attempts oscillate around the ad hoc selection of a small number of technology-driven rather than pathogenesis-driven variables, and the use of datasets possibly contaminated by high levels of noise and uninformative variables. These strategies also suffer from small patient populations, variable quality of clinical data, failure to integrate patient-derived variables, and the lack of demonstrated improved clinical outcomes on longterm followup.

\section{- HYPE}

In addition to the problems associated with current biomarkers, next-generation biomarkers present a number of unique challenges and risks. One such risk is that, being complex and costly to validate, it is tempting to evaluate them more leniently. However, in keeping with recommendations from the Institute of Medicine ${ }^{24}$, assays, choice of specimens, and criteria used for thresholds and variables selection during algorithm development need to be transparent and rigorously validated. We also need to assess their prognostic value and their ability to predict and impact on clinical outcomes. Finally, we must carefully review their appropriateness and pertinence for use in the clinical situation.

\section{The Example of Intestinal Microbiota: Availability versus Pathogenic Relevance}

Strong evidence suggests that gut microbiota influence our immune system ${ }^{25}$. In murine models of RA, dysimmunity does not develop in germ-free conditions. The impact of gut microbiota on the onset and progression of RA has been reported $^{26}$. Microbiome composition differentiates RA from non-RA patients, as well as early from established RA. Lay reports on these observations drive patients to try trendy dietetic interventions on intestinal microbiota to improve or even cure their arthritis. Is the science already in?

The colon has some of the highest observed densities of living organisms on earth ${ }^{14}$. Feces contain $10^{12}$ to $10^{13}$ bacteria per gram, but also large numbers of archaea, fungi, helminths, and perhaps an even larger number of viruses. For technical and conceptual reasons, bacteria remain the best studied at the moment. These bacteria thrive on undigested fibers, producing a variety of digestion products. The absorption of these products can contribute up to $6-10 \%$ of the total energy intake of the host.

Short chain volatile fatty acids such as acetic ( 2 carbons), propionic (3 carbons), and butyric (4 carbons) acids generated by the intestinal flora modulate gene expression in immune cells and other organs including bone and brain ${ }^{25}$. Butyrate exerts potent effects on immune cells in vitro and in animal models where it can shape the immune system by altering the epigenome ${ }^{27,28,29}$. However, the butyrate/host interaction is very complex to analyze in humans since it is influenced by host genetics, diet (source of undigested fibers), butyrate metabolism, and composition of gut microbiota. Butyrate may also have distinct impacts at physiologic versus pharmacologic concentrations ${ }^{28,30}$.

In humans, the composition of microbiota is modulated by age, mode of birth (cesarean vs vaginal), diet changes, diseases, antibiotic use (recent and remote), travel, and several other factors such as drugs, for example, metformin ${ }^{31}$. As a result, gut microbiota is highly variable between individuals and may change within a given individual over time.

Potential problems with this approach appear understated. First, we currently rely on the Koch's postulates stipulating that the prevalence of individual bacteria in a disease state is linked to pathogenesis. However, microbiome pathogenicity may depend on the interaction between multiple taxa. Alternatively, bacteria triggering the onset of immune disease may come and go before the clinical manifestations are recognized. Second, several drugs in common use are partly metabolized in the gut, potentially affecting their efficacy and toxicity as well as gut flora. It is thus important to take drugs into account when considering the correlations of microbiota with clinical outcomes. Third and most important, are stools the right specimen to study or are they studied merely due to ease of collection? Perhaps sampling bacteria residing in the mucus lining the epithelium, closer to immune cells, might be more relevant. Maybe we should study ileal bacteria, distinct from those in feces and more similar to the oral microbiome, since they are closer to Peyer's patches, where immune cells are matured and concentrated ${ }^{32}$.

In summary, simple associations of a disease with specific microbes are not sufficient to assume their significant role in pathogenesis.

\section{Specific Risks and Challenges of Next-generation Biomarkers}

The next-generation of biomarkers also have risks that come from their complexity and cost. Overfitting is definitively a risk, which can result in predictive models that lack reproducibility across cohorts, over an extended period of time, and under varying treatments. In addition, complex next-generation biomarkers often lend themselves to stratification into a large number of subsets, who may not fit into simplistic disease definitions based mostly on clinical grounds or conventional biomarkers. Their reliance on multiple parameters raises the potential for hidden co-correlations (e.g., microbiota and host genetics), making more difficult their combination with current or other next-generation biomarkers (MultiOmics), with clinical parameters, and with patient-related outcomes.

These complex biomarkers also constitute a potential threat to the healthcare system ${ }^{33}$. Indeed, their use might increase costs markedly, boosting ordering of additional tests

Personal non-commercial use only. The Journal of Rheumatology Copyright (c) 2019. All rights reserved. 
and referrals to specialists. Moreover, people with advanced training in statistics and computer science will be needed to perform quality control on a daily basis and ensure consistency of the testing. Without appropriate validation, their use may increase the number of patients considered at risk for a disease, without guarantees for improved clinical outcomes, and might even be detrimental due to the generated anxiety or to ill-oriented attempts at treatment. Finally, it may be tempting for promoters to use algorithms giving results most beneficial to marketing or commercial purposes rather than to patients ${ }^{33}$.

Ultimately, these biomarkers frequently require biospecimens that are not routinely collected, reducing the number of existing cohorts available to validate them. In many cases, their interpretation also depends on variables not available clinically or in administrative databases, such as host genetics and type of diet. As a consequence, there is a need for well-designed longterm observational cohorts with complete and high-quality clinical data and biomarker capacity, ideally from several geographic areas with genetic and environmental diversity ${ }^{34}$. The difficulty of funding these cohorts over the long term needs to be addressed. The alternative is hazardous introduction of incompletely validated tests into the clinical market.

Current biomarkers are relatively simple to use and understand, while generally very helpful. Nonetheless, biomarkers are often wrongly used, sometimes replacing the patient as objective of treatment. The CHOOSING WISELY campaign appropriately reminds us that careful clinical evaluation, both before and after the test, remains essential ${ }^{6}$.

Next-generation biomarkers resulting from the analysis of big data may certainly improve our understanding of disease pathogenesis and the prediction of treatment response, while informing on causal factors underlying disease progression. As such, they essentially help to subset patients into narrower more homogeneous groups, and are paving the way to personalized medicine tailored to individuals rather than groups.

Yet each next-generation biomarker needs to be evaluated very rigorously. The choice of techniques as well as the characteristics of biospecimens must be validated. Their prognostic value over current methods must be demonstrated, as well as their ability to predict the effects of interventions on clinical outcomes and help monitor the response to therapy. To attain these objectives, well-characterized cohorts followed over a long period are essential. Finally, we will need to evaluate the proposed uses of candidate next-generation biomarkers, to ensure that they are appropriate and result in improved outcomes.

The exciting prospects of next-generation biomarkers come with significant risks. It may be tempting for developers not to be transparent about their choices for thresholds and algorithms ${ }^{35}$. Past experience has shown that biomarkers may be manipulated in a way that the net result may be unfavorable despite increased $\operatorname{costs}^{36}$. Clinicians must play an essential validation role to optimize the use of next-generation biomarkers, even if very few are trained appropriately to understand and evaluate their strengths and limitations.

For sure, the next generation of biomarkers based on big data heralds a new, exciting, yet controversial era for rheumatology.

\section{REFERENCES}

1. Biomarkers Definitions Working Group. Biomarkers and surrogate endpoints: preferred definitions and conceptual framework. Clin Pharmacol Ther 2001;69:89-95.

2. Ballman KV. Biomarker: predictive or prognostic? J Clin Oncol 2015;33:3968-71.

3. FDA-NIH Biomarker Working Group. BEST (Biomarkers, EndpointS, and other Tools) Resource. Silver Spring, MD:FDA-NIH; 2016. [Internet. Accessed April 9, 2019.] Available from: www.ncbi.nlm.nih.gov/books/nbk326791

4. Mayr M, Zhang J, Greene AS, Gutterman D, Perloff J, Ping P. Proteomics-based development of biomarkers in cardiovascular disease: mechanistic, clinical, and therapeutic insights. Mol Cell Proteomics 2006;5:1853-64.

5. Vercellone J, Cohen L, Mansuri S, Zhang PL, Kellerman PS. Bartonella endocarditis mimicking crescentic glomerulonephritis with pr3-ANCA positivity. Case Rep Nephrol 2018;2018:9607582.

6. Cassel CK, Guest JA. Choosing wisely: helping physicians and patients make smart decisions about their care. JAMA 2012;307:1801-2.

7. Leblanc-Trudeau C, Dobkin PL, Carrier N, Cossette P, de Brum-Fernandes AJ, Liang P, et al. Depressive symptoms predict future simple disease activity index scores and simple disease activity index remission in a prospective cohort of patients with early inflammatory polyarthritis. Rheumatology 2015;54:2205-14.

8. Barra L, Pope JE, Orav JE, Boire G, Haraoui B, Hitchon C, et al. Prognosis of seronegative patients in a large prospective cohort of patients with early inflammatory arthritis. J Rheumatol 2014;41:2361-9.

9. Catrina AI, Svensson CI, Malmstrom V, Schett G, Klareskog L. Mechanisms leading from systemic autoimmunity to joint-specific disease in rheumatoid arthritis. Nat Rev Rheumatol 2017;13:79-86.

10. England BR, Thiele GM, Mikuls TR. Anticitrullinated protein antibodies: origin and role in the pathogenesis of rheumatoid arthritis. Curr Opin Rheumatol 2017;29:57-64.

11. Carrier N, Roux S, Masetto A, Fernandes A, Liang P, Maoui M, et al. The changing faces of rheumatoid arthritis patients at presentation: a 20-year study [abstract]. Arthritis Rheumatol 2018;70 Suppl 10:2983.

12. Nell-Duxneuner V, Machold K, Stamm T, Eberl G, Heinzl H, Hoefler E, et al. Autoantibody profiling in patients with very early rheumatoid arthritis: a follow-up study. Ann Rheum Dis 2010;69:169-74.

13. Shendure J, Balasubramanian S, Church GM, Gilbert W, Rogers J Schloss JA, et al. DNA sequencing at 40: past, present and future. Nature 2017;550:345-53.

14. Rowan-Nash AD, Korry BJ, Mylonakis E, Belenky P. Cross-domain and viral interactions in the microbiome. Microbiol Mol Biol Rev 2019;83:e00044-18.

15. See P, Lum J, Chen J, Ginhoux F. A single-cell sequencing guide for immunologists. Front Immunol 2018;9:2425.

16. Wang D, Eraslan B, Wieland T, Hallstrom B, Hopf T, Zolg DP, et al. A deep proteome and transcriptome abundance atlas of 29 healthy human tissues. Mol Syst Biol 2019;15:e8503.

17. Hammaker D, Firestein GS. Epigenetics of inflammatory arthritis. Curr Opin Rheumatol 2018;30:188-96.

Personal non-commercial use only. The Journal of Rheumatology Copyright (C 2019. All rights reserved. 
18. Kirschner M. Systems medicine: Sketching the landscape. Methods Mol Biol 2016;1386:3-15.

19. Plant D, Maciejewski M, Smith S, Nair N, Maximising Therapeutic Utility in Rheumatoid Arthritis Consortium - the RAMS Study Group, Hyrich K, et al. Profiling of gene expression biomarkers as a classifier of methotrexate nonresponse in patients with rheumatoid arthritis. Arthritis Rheumatol 2019 Jan 7 (E-pub ahead of print).

20. de Rotte MCFJ, Pluijm SMF, de Jong PHP, Bulatović Ćalasan M, Wulffraat NM, Weel AEAM, et al. Development and validation of a prognostic multivariable model to predict insufficient clinical response to methotrexate in rheumatoid arthritis. PLoS One 2018;13:e0208534.

21. Petty RE, Southwood TR, Manners P, Baum J, Glass DN, Goldenberg J, et al. International league of associations for rheumatology classification of juvenile idiopathic arthritis: second revision, Edmonton, 2001. J Rheumatol 2004;31:390-2.

22. Eng SW, Duong TT, Rosenberg AM, Morris Q, Yeung RS, REACCH Out, et al. The biologic basis of clinical heterogeneity in juvenile idiopathic arthritis. Arthritis Rheumatol 2014;66:3463-75.

23. Banchereau R, Hong S, Cantarel B, Baldwin N, Baisch J, Edens M, et al. Personalized immunomonitoring uncovers molecular networks that stratify lupus patients. Cell 2016;165:551-65.

24. Institute of Medicine. Evaluation of biomarkers and surrogate endpoints in chronic disease. Micheel CM, Ball JR, editors. Washington, DC: The National Academies Press; 2010.

25. Schroeder BO, Backhed F. Signals from the gut microbiota to distant organs in physiology and disease. Nat Med 2016; 22:1079-89.

26. Maeda Y, Takeda K. Role of gut microbiota in rheumatoid arthritis. J Clin Med 2017;6:e60.

27. Kim DS, Kwon JE, Lee SH, Kim EK, Ryu JG, Jung KA, et al.
Attenuation of rheumatoid inflammation by sodium butyrate through reciprocal targeting of HDAC2 in osteoclasts and HDAC8 in T cells. Front Immunol 2018;9:1525.

28. Knudsen K, Lærke H, Hedemann M, Nielsen T, Ingerslev A, Nielsen D, et al. Impact of diet-modulated butyrate production on intestinal barrier function and inflammation. Nutrients 2018;10:1499.

29. Macpherson AJ, de Aguero MG, Ganal-Vonarburg SC. How nutrition and the maternal microbiota shape the neonatal immune system. Nat Rev Immunol 2017;17:508-17.

30. Spina L, Cavallaro F, Fardowza N, Lagoussis P, Bona D, Ciscato C, et al. Butyric acid: Pharmacological aspects and routes of administration. Dig Liver Dis Suppl 2007;1:7-11.

31. Rinninella E, Raoul P, Cintoni M, Franceschi F, Miggiano GAD, Gasbarrini A, et al. What is the healthy gut microbiota composition? A changing ecosystem across age, environment, diet, and diseases. Microorganisms 2019;7:e14.

32. Villmones HC, Haug ES, Ulvestad E, Grude N, Stenstad T, Halland A, et al. Species level description of the human ileal bacterial microbiota. Sci Rep 2018;8:4736.

33. Mandl KD, Manrai AK. Potential excessive testing at scale: biomarkers, genomics, and machine learning. JAMA 2019 Feb 8 (E-pub ahead of print).

34. Miossec P, Verweij CL, Klareskog L, Pitzalis C, Barton A, Lekkerkerker F, et al. Biomarkers and personalised medicine in rheumatoid arthritis: a proposal for interactions between academia, industry and regulatory bodies. Ann Rheum Dis 2011;70:1713-8.

35. Redberg RF. The medicalization of common conditions. JAMA Intern Med 2016;176:1863.

36. Grady D, Redberg RF. Less is more: how less health care can result in better health. Arch Intern Med 2010;170:749-50. 